\title{
Teleconsultations between Patients and Healthcare Professionals in Primary Care in Catalonia: The Evaluation of Text Classification Algorithms Using Supervised Machine Learning
}

\author{
Francesc López Seguí 1,2 ${ }^{D}$, Ricardo Ander Egg Aguilar ${ }^{3}$, Gabriel de Maeztu ${ }^{4}$, \\ Anna García-Altés ${ }^{5}$, Francesc García Cuyàs ${ }^{6}$, Sandra Walsh ${ }^{7}$, Marta Sagarra Castro ${ }^{8}$ and \\ Josep Vidal-Alaball ${ }^{9,10, *(D)}$ \\ 1 TIC Salut Social-Ministry of Health, 08028 Barcelona, Spain; flopez@ticsalutsocial.cat \\ 2 CRES\&CEXS—Pompeu Fabra University, 08003 Barcelona, Spain \\ 3 Faculty of Medicine, Barcelona University, 08036 Barcelona, Spain; ricardo.anderegg@gmail.com \\ IOMED Medical Solutions, 08041 Barcelona, Spain; gabriel.maeztu@iomed.es \\ 5 Agency for Healthcare Quality and Evaluation of Catalonia (AQuAS), Catalan Ministry of Health, \\ 08005 Barcelona, Spain; agarciaaltes@gencat.cat \\ 6 Sant Joan de Déu Hospital, Catalan Ministry of Health, 08950 Barcelona, Spain; 31557fgc@gmail.com \\ 7 Institut de Biologia Evolutiva (UPF-CSIC), Pompeu Fabra University, 08003 Barcelona, Spain; \\ sandra.walsh34@gmail.com \\ 8 Centre d'Atenció Primària Capellades, Gerència Territorial de la Catalunya Central, Institut Català de la \\ Salut, 08786 Sant Fruitós de Bages, Spain; msagarra.cc.ics@gencat.cat \\ 9 Health Promotion in Rural Areas Research Group, Gerència Territorial de la Catalunya Central, \\ Institut Català de la Salut, 08272 Sant Fruitós de Bages, Spain \\ 10 Unitat de Suport a la Recerca de la Catalunya Central, Fundació Institut Universitari per a la recerca a \\ l'Atenció Primària de Salut Jordi Gol i Gurina, 08272 Sant Fruitós de Bages, Spain \\ * Correspondence: jvidal.cc.ics@gencat.cat
}

Received: 15 December 2019; Accepted: 7 February 2020; Published: 9 February 2020

\begin{abstract}
Background: The primary care service in Catalonia has operated an asynchronous teleconsulting service between GPs and patients since 2015 (eConsulta), which has generated some 500,000 messages. New developments in big data analysis tools, particularly those involving natural language, can be used to accurately and systematically evaluate the impact of the service. Objective: The study was intended to assess the predictive potential of eConsulta messages through different combinations of vector representation of text and machine learning algorithms and to evaluate their performance. Methodology: Twenty machine learning algorithms (based on five types of algorithms and four text representation techniques) were trained using a sample of 3559 messages $(169,102$ words) corresponding to 2268 teleconsultations (1.57 messages per teleconsultation) in order to predict the three variables of interest (avoiding the need for a face-to-face visit, increased demand and type of use of the teleconsultation). The performance of the various combinations was measured in terms of precision, sensitivity, F-value and the ROC curve. Results: The best-trained algorithms are generally effective, proving themselves to be more robust when approximating the two binary variables "avoiding the need of a face-to-face visit" and "increased demand" (precision $=0.98$ and 0.97 , respectively) rather than the variable "type of query" (precision $=0.48$ ). Conclusion: To the best of our knowledge, this study is the first to investigate a machine learning strategy for text classification using primary care teleconsultation datasets. The study illustrates the possible capacities of text analysis using artificial intelligence. The development of a robust text classification tool could be feasible by validating it with more data, making it potentially more useful for decision support for health professionals.
\end{abstract}

Keywords: machine learning; teleconsultation; primary care; remote consultation; classification 


\section{Introduction}

eConsulta is an asynchronous teleconsultation service between patients and GPs as part of the electronic health records of the public primary healthcare system of Catalonia. In operation since the end of 2015, this secure messaging service was designed to complement face-to-face consultations with primary healthcare teams (PHT). It was gradually implemented up until 2017, when the service became available to every PHT; currently, all of them have used this tool at least once.

An earlier study analysed the reasons why patients sought a consultation, which resulted in a patient-doctor interaction, as well as the subjective perception of the GP if they avoided a face-to-face visit or if it led to a consultation which otherwise would not have occurred, by means of a retrospective review of text messages relating to each case [1]. The results show there was a broad consensus among GPs that eConsulta has the potential to resolve patient queries (avoiding the need for a face-to-face visit in $88 \%$ of cases) for every type of consultation. In addition, GPs declared that ease of access led to an increase in demand (queries which otherwise would not have been made) in $28 \%$ of cases. Therefore, the possibility of eConsulta replacing a conventional appointment stands at between $88 \%$ and $63 \%$ $(88 \% \times(1-28 \%))$. The most common use of e-consultation was for the management of test results $(35 \%)$, clinical enquiries $(16 \%)$ and the management of repeat prescriptions $(12 \%)$.

Technology offers new possibilities for policy evaluation in conjunction with the aforementioned classical approaches. Artificial intelligence tools are already widely used in the field of healthcare in areas such as the prediction and management of depression, voice recognition for people with speech impediments, the detection of changes in the biopsychosocial status of patients with multiple morbidities, stress control, the treatment of phantom limb pain, smoking cessation, personalized nutrition by prediction of glycaemic response, to try to detect signs of depression and in particular for reading medical images [2-6]. The generation of data implies a huge potential for the impact assessment of these interventions with new analytical tools.

The classification of texts in the medical field has also been used to conduct a review of influenza detection and prediction through social networking sites [7-9] and in the analysis of texts from internet forums $[10,11]$. More specifically, in the framework of teleconsultations, a US-based study used machine learning to annotate 3000 secure message threads involving patients with diabetes and clinical teams according to whether they contained patient-reported hypoglycaemia incidents [12]. As far as the authors are aware, no study has looked into the development of a text classification algorithm in the context of teleconsultations between patients and primary care physicians.

The present study aims to evaluate specific text classification algorithms for eConsulta messages and to validate their predictive potential. The algorithms have been trained using a vector representation of text from the body of the message and the three variable annotations that primary healthcare professionals in Central Catalonia used in a previous study: avoiding the need for a face-to-face visit, increased demand and type of use of the teleconsultation [1]. Our study represents an exhaustive exploratory analysis of text classification algorithms of teleconsultation messages between GPs and patients that can provide useful information for future research and a potential use for decision support in healthcare.

\section{Methodology}

\subsection{Data Acquisition}

The teleconsultations that had previously been classified that were used as the basis for training the algorithm are those which were acquired in the study by a previous study (López) (Table 1). They are part of the health records of the Gerència Territorial de la Catalunya Central of the Institut Català de la Salut covering the period from when the tool was first used until the date of its extraction for analysis purposes (8 April 2016 to 18 August 2018). Message deidentification was performed by substituting all 
possible names contained in the Statistical Institute of Catalonia database [13] with a common token and removing all other personal attributes. The classification method used for the conversations is described and justified by López et al. 2019: Every healthcare professional who received an eConsulta labelled it according to whether, in their opinion, it avoided the need for a face-to-face consultation, led to an increased demand and by type of teleconsultation (Appendix A.1). These results of this annotation, with the corresponding messages, were used to train the text classification model using the three variables previously mentioned (Table 2).

Table 1. Data recorded by the teleconsulting system.

\begin{tabular}{lccccc}
\hline Conversation Title & Conversation ID & Message ID & From & To & Message \\
\hline $\begin{array}{c}\text { Travelling to } \\
\text { Australia }\end{array}$ & C1 & M1 & Mr. John Patient & Ms. Jane Doctor & $\begin{array}{c}\text { Dear doctor, I'm travelling to } \\
\text { Australia on 15 December. Do I } \\
\text { need to have any vaccinations? } \\
\text { Many thanks }\end{array}$ \\
\cline { 2 - 5 } & M2 & Ms. Jane Doctor & Mr. John Patient & $\begin{array}{c}\text { Hi, vaccination is required for } \\
\text { travel to Australia }\end{array}$ \\
\hline
\end{tabular}

Table 2. Annotation by the GP.

\begin{tabular}{cccc}
\hline Conversation ID & Face-to-Face Visit Avoided? & Increased Demand? & Type of Visit \\
\hline C1 & Yes & No & 6 (Vaccinations) \\
\hline
\end{tabular}

Most of the data were received with a tabular arrangement, and the texts and their labels were in different files that were merged according to the Conversation ID. The data cleaning was a multi-step process. Regarding the text: First, all the tokens of anonymized names were changed to a standard name of the country "Juan". The title was merged with the body of the message, adding the token "xxti" before the title and "tixx" after the title; that way we would not lose the information that this was the title. The texts were all converted to lowercase, and we extracted the length (in words and in characters) of every message to use as extra independent variables. As additional variables, the day of the month and time of the day were extracted from the date of the message.

\subsection{Vector Representation of Text in eConsulta Messages}

The emails needed to be represented in some way in order to use them as input for the models. A common practice in machine learning is the vector representation of words. These vectors capture hidden information about the language, such as word analogies and semantics, and improve the performance of text classifiers.

Four techniques have been used to generate the vector representation of texts. The Bag of Words (BoW) approach counts the number of times pairs of words appear in each document. The document is represented as a vector of a finite vocabulary. The Term Frequency-Inverse Document Frequency (TF-IDF) method assigns paired words a weight depending on the number of times they appear in a particular document (the Term Frequency), while discounting its frequency in other documents (Inverse Document Frequency): The more documents a word appears in, the less valuable that word is as a signal to differentiate any given document. Word2Vec is a two-layered neuronal network that trains and processes text. Its input is a corpus of text and its output is a set of vectors for the words in the corpus, with words represented by numbers. The initial vector assigned to a word cannot be used to accurately predict its context, meaning its components must be adjusted (trained) through the contexts in which they are found. In this way, repeating the process for each word, word vectors with similar contexts end up in nearby vector spaces. Fasttext [14] is used to obtain word2vec vectors. Finally, the objective of Doc2vec is to create a numerical representation of a document, regardless of its length. This approach represents each document by a dense vector, which learns to predict the words in the document [15]. In all cases, before carrying out the vectorization of the texts, these were first 
tokenized and any stop-words eliminated (those which are taken to have no meaning in their own right, such as articles, pronouns or prepositions).

In each instance, the vectors were enriched by supplementing them with similar texts in Catalan and Spanish [16]. The external data used to enrich the corpus were models of interactions extracted from online databases with colloquial language similar to that used in eConsulta. Where augmented BOW, TF-IDF and Word2Vec were used, word and character length and word density were also used as predictor variables.

\subsection{Training and Testing AI Algorithms}

The task addressed in this study is a multiclass classification with respect to the type of visit and two binary classifications for the other two variables (avoiding visit and increased demand). For each text vector representation algorithm five different algorithms were implemented: Random Forest, Gradient Boosting (lightGBM), Fasttext, Multinomial Naive Bayes and Naive Bayes Complement [17]. Bayesian text classifiers are the most standard algorithms in this setting. A convolutional neural network was also used using the augmented Word2vec vectors. We tested the performance of the algorithms through a stratified 10-fold cross-validation: During 10 iterations/trainings, 9 divisions served as learning and 1 as a test.

The coefficients of interest to evaluate the goodness of the algorithms were precision (the fraction of relevant instances between the retrieved instances/proportion of correct predictions of the total of all predicted cases) and sensitivity (the number of correct classifications for the positive class "true positive"). It was decided not to use the "accuracy" coefficient since it is a metric that, given an unbalanced dataset like the one under investigation, can result in a very high score in spite of the fact that the classifier works poorly, since it assesses the number of total hits without taking into account whether most of the data is of the same class. The F value is used to determine a weighted single value of accuracy and completeness. The diagnostic value is assessed by means of the ROC curve. The goodness-of-fit of all the coefficients is represented as a value between 0 and 1 .

Python 3.7 and the following libraries were used for the algorithm training: numpy [18], matplotlib [19], seaborn [20], altair [21], scikit-learn [22], pandas [23], gensim [24], nltk [25], fasttext [14], pytorch [26] and lightGBM [27]. The majority of the code was carried out on Jupyter Notebooks [28].

\subsection{Ethical Considerations}

The study was approved by the Ethical Committee for Clinical Research at the Foundation University Institute for Primary Health Care Research Jordi Gol and Gurina, registration number P19/096-P, and carried out in accordance with the Declaration of Helsinki [29].

\section{Results}

In order to assess the predictive potential of eConsulta messages regarding the three variables of interest, we first aimed to identify the best combination of algorithms. A total of 3559 messages (169,102 words) corresponding to 2268 teleconsultations (1.57 messages per teleconsultation) were analysed in a framework of 20 different combinations of vector representation of text and machine learning algorithms (Table 3). We assessed the performance of the combinations of algorithms though a stratified 10-fold cross-validation analysis. Figure 1 shows the performance of the most stable algorithm (best metrics, in general) according to the predictor variable. 
Table 3. Text representations and algorithms used.

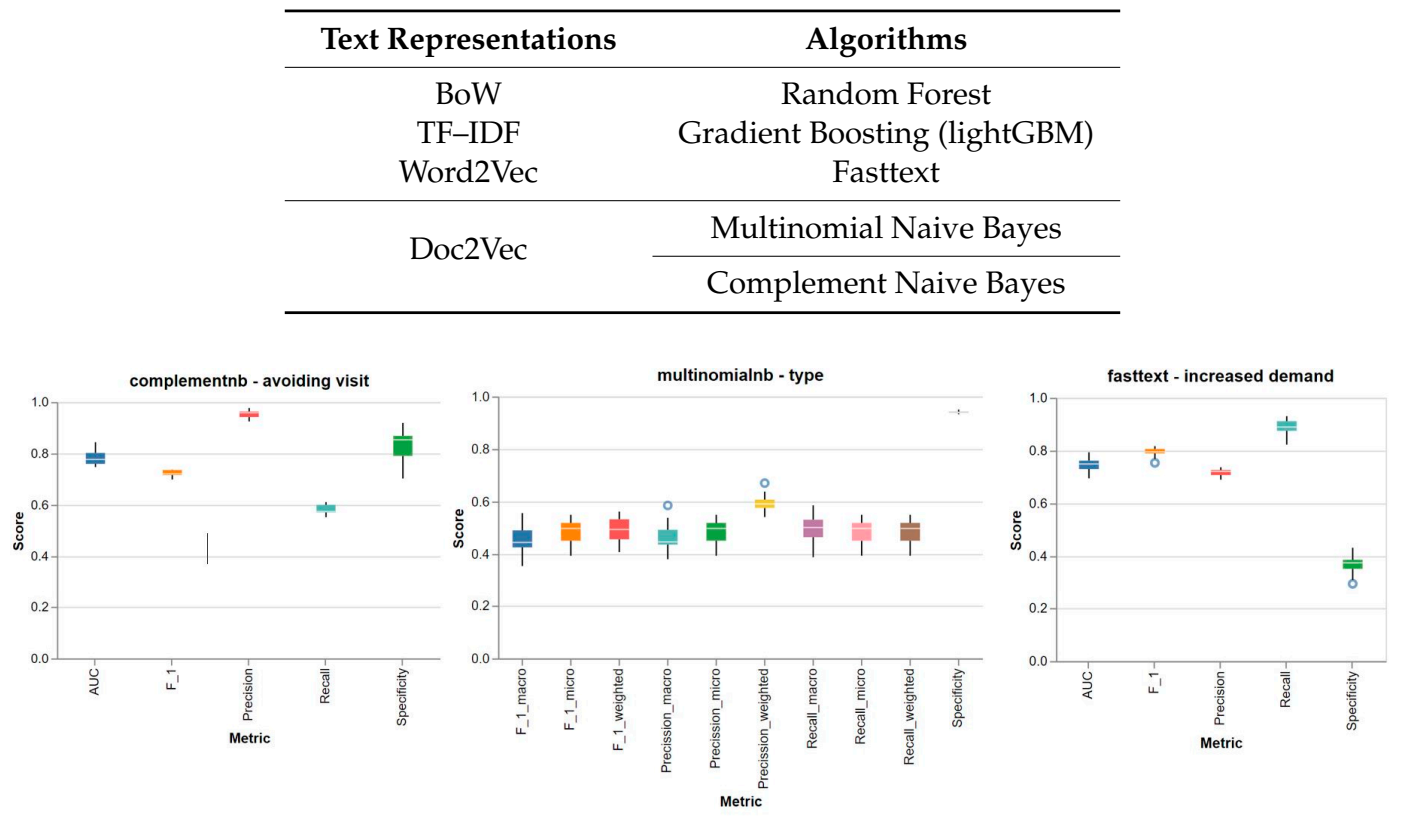

Figure 1. Performance metrics of algorithms.

Specific combinations of algorithms per variable generally perform very well. Table 4 shows the evaluation metrics (mean + standard deviation of the 10 iterations) of the combination of algorithm and numerical representation of the text which has a better performance for each target variable. For all of the cases, the vectors obtained directly from the original texts have been more useful than those enriched with external texts. Table 4 shows that algorithms are generally effective, showing they are better when approximating the two binary variables (avoiding the need for a face-to-face visit, increased demand) than the variable "type of query". Thus, eConsulta's classifiers have a promising and robust predictive value, especially for binary variables.

Table 4. Results of the best algorithm/text representation combination, according to the variable to be approximated. Average (SD) of the 10 iterations.

\begin{tabular}{ccccc}
\hline Variable & Precision & Recall & F1 & Roc_AUC \\
\hline \multirow{3}{*}{ Avoiding the need of a face-to-face visit } & Random Forest & FastText & FastText & ComplementNB \\
& TF-IDF & Word2Vec & Word2Vec & TF-IDF \\
& $0.98(0.026)$ & $0.99(0.005)$ & $0.92(0.004)$ & $0.79(0.032)$ \\
\hline \multirow{2}{*}{ Increased demand } & Random Forest & FastText & FastText & FastText \\
& TF-IDF & Word2Vec & Word2Vec & Word2Vec \\
& $0.97(0.057)$ & $0.89(0.029)$ & $0.79(0.018)$ & $0.75(0.031)$ \\
\hline \multirow{2}{*}{ Type of use of the teleconsultation } & MultinomialNB & MultinomialNB & MultinomialNB \\
(micro averaged score) & BOW & BOW & BOW & \\
& $0.48(0.049)$ & $0.48(0.049)$ & $0.48(0.049)$ & \\
\hline
\end{tabular}

As a whole, the results illustrate eConsulta's algorithm classifiers potential predictive value and provide a valuable insight into the implementation of AI methodologies for healthcare teleconsultation.

\section{Discussion}

\section{Limitations}

Several limitations apply to this study and the results must be understood in light of these shortcomings. First, our classifier is restricted to one dataset and the training set was relatively small. 
Although the study used all the available information, more data is needed to generalize the model and avoid overfitting.

The amount of data with which the algorithms were tested is especially relevant in the case of trying to calculate the variable "type of message", since the number of types which contain the classification [13], meaning the quantity of messages of each with which the classification algorithm has been trained, is minimal, thus diminishing its predictive capacity. This may have had implications to our approach and subsequent results. What is required is not only more messages, they must also contain as much information as possible. Validating the algorithm requires a replication of the proposed methodology with a larger data set, together with the analysis of subgroups. Likewise, the goodness of fit of the results may be caused by overfitting: The model explains this set of data well, but could show weaknesses when generalizing to others, limiting its potential for extrapolation. Because of that, this study includes exhaustive detail of the methodology used in order that it can be replicated.

Second, an error analysis was not conducted. This analysis might have helped us to understand why certain posts where misclassified or classified correctly.

Using complex mathematical models makes it difficult to explain why some work better than others. The vectors would need to be evaluated at a lower level in order to have a better idea as to which characteristics redirect the model towards one decision or another. This analysis is of interest for future applications of these techniques on a larger scale or for applications related to medical practice.

\section{Conclusions}

In Catalonia, the number of conversations and messages now stand at approximately 370,000 and 500,000, respectively. Applying a classification algorithm like the one proposed here would help us understand the nature of the conversations and their impact in real time. Future research should evaluate the use of automation (to send a diagnostic test, generate an alert or "thank you" and close the case) as a tool for decision support for healthcare professionals to improve the management of clinical cases and to save GPs time. Natural Language Processing approaches should further analyse the content of the teleconsultations and proactively offer clinicians agile resources to deal with the cases.

This article has shown that the implementation of an algorithm for the prediction of factors such as a reduction in the number of face-to-face visits, induced demand or type of consultation is technically feasible and potentially useful in the context of service planning, management of the demand and evaluation. This study presents a combination of algorithms based on machine learning and a more efficient representation of vectors for this type of data. This study is an initial exploration into the potential of teleconsultation and the promising use of artificial intelligence for the evaluation of digital health interventions.

Author Contributions: Conceptualization, F.L.S., J.V.-A.; methodology, R.A.E.A., G.d.M.; software, R.A.E.A., G.d.M., S.W.; validation, F.L.S., R.A.E.A., G.d.M., A.G.-A., F.G.C., S.W., M.S.C., J.V.-A.; formal analysis, F.L.S., R.A.E.A., J.V.-A.; investigation, F.L.S., R.A.E.A., G.d.M., S.W., J.V.-A.; resources, F.L.S., J.V.-A.; data curation, R.A.E.A.; writing-original draft preparation, F.L.S., J.V.-A.; writing-review and editing, F.L.S., R.A.E.A., G.d.M., A.G.-A., F.G.C., S.W., J.V.-A.; visualization, F.L.S., A.G.-A., F.G.C., S.W., M.S.C., J.V.-A.; supervision, F.L.S., A.G.-A., F.G.C., M.S.C., J.V.-A.; project administration, F.L.S.; funding acquisition, F.L.S. All authors have read and agreed to the published version of the manuscript.

Acknowledgments: This study was conducted with the support of the Secretary of Universities and Research of the Department of Business and Knowledge at the Generalitat de Catalunya. We would like to thank the staff at the Technical and Support Area of Gerència Territorial de la Catalunya Central for their assistance during the data collection phase.

Conflicts of Interest: The authors declare no conflict of interest. 


\section{Abbreviations}

$\begin{array}{ll}\text { GP } & \text { General Practitioner } \\ \text { BOW } & \text { Bag of Words } \\ \text { TF-IDF } & \text { Term Frequency-Inverse Document Frequency } \\ \text { ROC } & \text { Receiver Operating Characteristics }\end{array}$

\section{Appendix A}

Reasons for using eConsulta

\section{Appendix A.1 Administrative}

1 Management of test results

The patient provides the results of tests carried out in an external centre in order that they are recorded in their medical history.

The GP provides the results of tests with normal results.

The GP deals with questions related to tests requested by the patient.

The GP requests tests after conducting a follow-up teleconsultation.

2 Temporary disability management

The patient communicates changes to their health related to an upcoming temporary disability.

The GP tracks the progress of a temporary disability in conjunction with face-to-face visits.

3 Management of visits/referrals

$0 \quad$ The patient has an enquiry which the GP thinks ought to be dealt with by a specialist and refers them. They can also report incidents resulting from any referrals made.

The GP resolves incidents relating to the timing of visits.

- The GP cancels visits from other clinicians in cases in which the problem has been resolved following completion of the e-consultation.

$\checkmark \quad$ Validation of appointments with other specialists where the citizen needs more information about the motivation of the appointment.

4 Request for a clinical report/sick-note

The patient asks for a report/sick-note while consulting their medical history.

The GP asks the patient for more information in order to prepare the report.

5 Repeat prescriptions

The patient asks for their prescription to be updated if it has been modified by an external specialist, either because they do not use it or because it has expired.

The GP warns the patient that their prescription is about to expire and updates it.

The GP cancels an unnecessary prescription following an e-consultation.

6 Vaccinations

Updates of immunization schedules and general enquiries regarding vaccinations.

Questions concerning vaccinations for travel overseas.

7 Other administrative issues: Any administrative procedure which can be resolved without being physically present.

\section{Appendix A.2 Medical}

8 Medical enquiries: The patient has a question about their health that can be resolved without a physical examination. They can also attach photographs to accompany the description.

9 Issues regarding medicines: the patient asks a question about a prescription.

10 Questions regarding anticoagulants and dosage.

\section{Appendix A.3 Others}

11 Messages sent in error: The patient made a mistake.

12 Other.

13 Test messages. 


\section{References}

1. López Seguí, F.; Vidal Alaball, J.; Sagarra Castro, M.; García Altés, A.; García Cuyàs, F. Does teleconsultation reduce face to face visits? Evidence from the Catalan public primary care system. JMIR Prepr. 2019. [CrossRef]

2. Triantafyllidis, A.K.; Tsanas, A. Applications of Machine Learning in Real-Life Digital Health Interventions: Review of the Literature. JMIR 2019, 21, e12286. [CrossRef] [PubMed]

3. Luo, W.; Phung, D.; Tran, T.; Gupta, S.; Rana, S.; Karmakar, C.; Shilton, A.; Yearwood, J.; Dimitrova, N.; Ho, T.B.; et al. Guidelines for Developing and Reporting Machine Learning Predictive Models in Biomedical Research: A Multidisciplinary View. JMIR 2016, 18, e323. [CrossRef] [PubMed]

4. $\quad$ Li, Z.; Keel, S.; Liu, C.; He, Y.; Meng, W.; Scheetz, J.; Lee, P.Y.; Shaw, J.; Ting, D.; Wong, T.Y.; et al. An Automated Grading System for Detection of Vision-Threatening Referable Diabetic Retinopathy on the Basis of Color Fundus Photographs. Diabetes Care. 2018, 41, 1-8. [CrossRef] [PubMed]

5. Gulshan, V.; Peng, L.; Coram, M. Development and validation of a deep learning algorithm for detection of diabetic retinopathy in retinal fundus photographs. JAMA 2016, 316, 2402-2410. [CrossRef] [PubMed]

6. Vidal-Alaball, J.; Dídac Royo, F.; Zapata, M.A.; Gomez, F.M.; Fernandez, O.S. Artificial Intelligence for the Detection of Diabetic Retinopathy in Primary Care: Protocol for Algorithm Development. JMIR Res. Protoc. 2019, 8, e12539. [CrossRef] [PubMed]

7. Alessa, A.; Faezipour, M. Preliminary Flu Outbreak Prediction Using Twitter Posts Classification and Linear Regression with Historical Centers for Disease Control and Prevention Reports: Prediction Framework Study. JMIR Public Health Surveill. 2019, 5, e12383. [CrossRef] [PubMed]

8. Xu, S.; Markson, C.; Costello, K.L.; Xing, C.Y.; Demissie, K.; Llanos, A.A. Leveraging Social Media to Promote Public Health Knowledge: Example of Cancer Awareness via Twitter. JMIR Public Health Surveill. 2016, 2, e17. [CrossRef] [PubMed]

9. Doan, S.; Ritchart, A.; Perry, N.; Chaparro, J.D.; Conway, M. How Do You \#relax When You're \#stressed? A Content Analysis and Infodemiology Study of Stress-Related Tweets. JMIR Public Health Surveill. 2017, 3, e35. [PubMed]

10. McRoy, S.; Rastegar-Mojarad, M.; Wang, Y.; Ruddy, K.J.; Haddad, T.C.; Liu, H. Assessing Unmet Information Needs of Breast Cancer Survivors: Exploratory Study of Online Health Forums Using Text Classification and Retrieval. JMIR Cancer 2018, 4, e10. [CrossRef] [PubMed]

11. Bobicev, V.; Sokolova, M.; El Emam, K.; Jafer, Y.; Dewar, B.; Jonker, E.; Matwin, S. Can Anonymous Posters on Medical Forums be Reidentified? JMIR 2013, 15, e215. [CrossRef] [PubMed]

12. Chen, J.; Lalor, J.; Liu, W.; Druhl, E.; Granillo, E.; Vimalananda, V.G.; Yu, H. Detecting Hypoglycemia Incidents Reported in Patients' Secure Messages: Using Cost-Sensitive Learning and Oversampling to Reduce Data Imbalance. JMIR 2019, 21, e11990. [CrossRef] [PubMed]

13. IDESCAT. Noms de la Població. Available online: http://www.idescat.cat/noms/ (accessed on 24 September 2019).

14. Bojanowski, P.; Grave, E.; Jouilin, A.; Mikolov, T. Enriching word vectors with subword information. Trans. Assoc. Comput. Linguist. 2017, 5, 135-146. [CrossRef]

15. Le, Q.; Tomas, M. Distributed representations of sentences and documents. In Proceedings of the International Conference on Machine Learning, Beijing, China, 21-26 June 2014.

16. Ljubesic, N.; Toral, A. caWaC-A web corpus of Catalan and its application to language modeling and machine translation. LREC 2014, L14-1647, 1728-1732.

17. Rennie, J.D.; Shih, L.; Teevan, J.; Karger, D.R. Tackling the poor assumptions of naive bayes text classifiers. In Proceedings of the 20th International Conference on Machine Learning (ICML-03), Washington, DC, USA, 21-24 August 2003.

18. Van Der Walt, S.; Chris Colbert, S.; Varoquaux, G. The NumPy array: A structure for efficient numerical computation. Comput. Sci. Eng. 2011, 13, 22. [CrossRef]

19. Hunter, J.D. Matplotlib: A 2D Graphics Environment. Comput. Sci. Eng. 2007, 9, 9-95. [CrossRef]

20. mwaskom/seaborn: v0. 9.0. Available online: https://zenodo.org/record/1313201 (accessed on 30 January 2020).

21. Altair. Available online: https://altair-viz.github.io/index.html (accessed on 8 February 2020). 
22. Pedregosa, F.; Grisel, O.; Blondel, M.; Prettenhofer, P.; Weiss, R.; Dubourg, V.; Vanderplas, J.; Passos, A.; Cournapeau, D. Scikit-learn: Machine Learning in Python. JMLR 2011, 12, 2825-2830.

23. McKinney, W. Data structures for statistical computing in python. In Proceedings of the 9th Python in Science Conference, Austin, TX, USA, 9-15 July 2010; Volume 445.

24. Rehurek, R.; Sojka, P. Software framework for topic modelling with large corpora. In Proceedings of the LREC 2010 Workshop on New Challenges for NLP Frameworks, Valetta, Malta, 22 May 2010.

25. Bird, S.; Loper, E.; Klein, E. Natural Language Processing with Python; O'Reilly Media Inc.: Newton, MA, USA, 2009.

26. Paszke, A. Automatic differentiation in pytorch. In Proceedings of the 31st Conference on Neural Information Processing Systems (NIPS 2017), Long Beach, CA, USA, 4-9 December 2017.

27. Ke, G.; Meng, Q.; Finley, T.; Wang, T.; Chen, W.; Ma, W.; Ye, Q.; Liu, T. Lightgbm: A highly efficient gradient boosting decision tree. In Proceedings of the 31st Conference on Neural Information Processing Systems (NIPS 2017), Long Beach, CA, USA, 4-9 December 2017.

28. Kluyver, T.; Ragan-Kelley, B.; Pérez, F.; Granger, B.; Bussonnier, M.; Frederic, J.; Kelley, K.; Hamrick, J.; Grout, J.; Corlay, S.; et al. Jupyter Notebooks-a publishing format for reproducible computational workflows. ELPUB 2016. [CrossRef]

29. World Medical Association. World Medical Association Declaration of Helsinki. Ethical Principles for Medical Research Involving Human Subjects Helsinki. 2013. Available online: https:/www.wma.net/whatwe-do/medical-ethics/declaration-of-helsinki/ (accessed on 27 January 2020).

(C) 2020 by the authors. Licensee MDPI, Basel, Switzerland. This article is an open access article distributed under the terms and conditions of the Creative Commons Attribution (CC BY) license (http://creativecommons.org/licenses/by/4.0/). 